\title{
State entry regulation and home health agency quality ratings
}

\author{
Robert L. Ohsfeldt ${ }^{1}$ (D) Pengxiang Li $\mathbf{L i}^{2,3}$
}

\begin{abstract}
There is a substantial literature assessing the impact of entry restrictions created by state certificate-of-need (CON) programs on hospital and nursing home markets, but comparatively little research has focused on CON for home health agencies (HHAs). We assessed the impact of state CON programs for HHAs, and for potential substitute service providers, on quality ratings for HHAs. HHA quality ratings were obtained from the Home Health Compare database developed by the Centers for Medicare and Medicaid Services (CMS) for the last quarter of 2010 through the last quarter of 2013. The HHA-level data were augmented with county-level area characteristics for each HHA in the CMS database. An ordered logit model was used to estimate the association between state CON restrictions and Low, Medium, and High quality categories, adjusted for HHA and area characteristics. The results indicated that HHAs in states with CON for HHAs were less likely to have High quality ratings, and more likely to have Medium quality ratings, compared to agencies in states without CON for home health. Additional research is needed to assess whether the apparent adverse impact of CON on HHA quality is related to diminished competition among HHAs in states with CON.
\end{abstract}

Keywords Home health · Barriers to entry · Certificate of Need · Quality

$凶 \quad$ Robert L. Ohsfeldt

rohsfeldt@tamu.edu

Pengxiang Li

penli@pennmedicine.upenn.edu

1 School of Public Health, Texas A\&M University, 1266, TAMU, College Station, TX 77843-1266, USA

2 Perelman School of Medicine, University of Pennsylvania, Philadelphia, PA 19104, USA

3 Leonard Davis Institute of Health Economics, University of Pennsylvania, Philadelphia, PA, USA 
JEL Classification I18 $\cdot$ L51

\section{Introduction}

In the United States, there has been a trend over the past several decades toward moving the provision of health care services associated with long-term or short-term disability from institutional settings to patients' homes, provided by home health service providers through home health service agencies (David and Polsky 2014). This trend was fueled by an expectation that provision of certain services in a patient's home would be less costly than providing the same services in an institutional setting (David and Polsky 2014), which was reinforced by prevailing preference among patients to receive such services at home (Freeman 1995). As a result, expenditures on home health services in the United States, which were $\$ 15.1$ billion in 1991, grew to $\$ 88.8$ billion in 2015 (CMS 2017). This represents an annual growth rate of 7.3\%; substantially greater than the $5.8 \%$ annual growth in total healthcare expenditures over the same period.

A growing share of total home health services expenditures has been paid by Medicaid and Children's Health Insurance Program (CHIP) programs, which are funded by state government tax revenues with additional funding from the federal government. Specifically, the Medicaid/CHIP share of total home health expenditures increased from $16 \%$ in 1991 to $36 \%$ in 2015 , with a $10.8 \%$ annual growth rate over this period. Over time, the rapid growth in Medicaid spending stressed state government budgets, which led them to adopt various initiatives to attempt to control Medicaid spending growth (Tudor 1995; Wiener et al. 1999). Among these initiatives was the expansion of state "certificate-of-need" (CON) regulations, which traditionally had focused on controlling expansions in hospital capacity, to also target types of service providers accounting for substantial shares of Medicaid spending, such as nursing home services. The idea was that limiting the supply of nursing home services would limit the use of nursing home services by Medicaid recipients, which was assumed to result in lower expenditure growth (Ching 2015). By 2000, 37 states had CON for nursing home facilities, and 17 states had CON for home health agencies (HHAs). However, since 2000 there has been no change in state CON regulations, with the exception of New Hampshire, where CON was repealed in 2016 (National Conference of State Legislatures 2016).

There is a substantial literature focused on assessing the impact of state CON programs on healthcare costs, as well as access to and quality of care. However, most of this literature focuses on state $\mathrm{CON}$ programs for hospitals or nursing home facilities. Comparatively little research has focused on the effects of home health CON. The purpose of this paper is to assess the impact of state CON programs for HHAs, and for potential substitute service providers, on quality ratings for HHAs. The conceptual basis for this analysis rests on past research suggesting CON increases structural concentration in regulated health care service markets, coupled with past research suggesting greater competition among health care service providers improves service quality. The results of our empirical model indicated that HHAs in states with CON for home health were less likely to have high quality ratings, and more likely to have 
medium quality ratings, compared to agencies in states without $\mathrm{CON}$ for home health, with no statistically significant difference in the likelihood of low quality ratings.

\section{Background}

\subsection{CON regulation}

Hospital CON programs initially were envisioned as a means to address an incentive issue embedded in the "cost-based" hospital payment systems used by Medicare and most private payers in the 1970s, whereby hospitals could in effect increase payment rates for services they provided by expanding their capital base. CON regulation, by requiring hospitals to submit an application to a state health planning agency to demonstrate a need for expanded capital capacity, enabled states to block capital expansion by rejecting applications deemed unnecessary. The National Health Planning and Resources Development Act (or "Health Planning Act") of 1974 incentivized states to adopt hospital CON, and by 1980 all but 1 state had CON in place. However, Medicare replaced its cost-based payment with the inpatient prospective payment system (IPPS), which was fully implemented in 1986. The Health Planning Act was repealed in 1986, in part because IPPS eliminated the connection between hospital capital costs and Medicare payments. Subsequently, several states-mainly those with above-average population growth-eliminated hospital CON, but most states retained nursing home CON (see Ohsfeldt and Schneider 2006, Ch. 2).

An ex post rationale for hospital $\mathrm{CON}$ regulation emerged later, based on the observed "volume-outcome" relationship for complex surgical procedures, coupled with the potential for CON to concentrate volume for such procedures over a limited number of providers (e.g., Vaughan-Sarrazin et al. 2002). Assuming this rationale is valid, it would not seem to apply to relatively low-skilled home health services, or for nursing home facilities. Indeed, the original CON rationale - to control unnecessary capital expenditures - does not apply at all to a labor-intensive product such as home health services (Polsky et al. 2014).

Another explanation for the retention of state hospital CON, and the expansion of CON to other types of service providers, relates to the economic theory of regulation (Stigler 1971), whereby incumbent service providers use CON as a barrier to entry by potential competitors. This demand for $\mathrm{CON}$ by incumbent providers often coincides with the interests of state governments, in their role as Medicaid payers, to use CON as an indirect mechanism for rationing services for Medicaid enrollees, especially for services representing a substantial contribution to total Medicaid expenditures, such as nursing home care (Harrington et al. 2004; Wiener et al. 1999). Given the growing Medicaid payer share for home health services, a plausible objective of home health $\mathrm{CON}$ is to attempt to limit home health service capacity to restrict growth in Medicaid spending on home health. However, as Polsky et al. (2014) note, home health CON restricts entry by new HHAs, but does not limit the ability of existing HHAs to expand service capacity by hiring additional home health service workers. Thus, it is unclear if CON for HHAs entails a binding constraint on market-level home health service capacity. However, to the extent CON for HHAs is binding on the number of HHAs, 
it will increase structural market concentration, which may translate into reduced competition among HHAs in the home health service market.

\subsection{Competition and quality}

Economic theory predicts that competition among health care service providers will increase product quality if quality is at least partially observable (Pauly 2004). There is some literature to suggest that consumers (Phillips et al. 2007) or payers (Robinson and MacPherson 2012) use published facility-level quality information when selecting facilities to use or include in preferred networks, if the information is easy to absorb and focused on identifying the "best and worst" quality providers. Though mixed, the literature suggests that competition among hospitals is associated with improvement in several observable indicators of hospital quality (Bloom et al. 2015; FTC and DOJ 2014; Gaynor and Vogt 2000; Gutacker et al. 2016). Similarly, greater competition among nursing home providers tends to be associated with higher quality (Grabowski and Hirth 2003; Grabowski and Town 2011; Huang and Hirth 2016). To our knowledge, Polsky et al. (2014) is the only published study that has examined the impact of competition among HHAs on service quality. Using Medicare claims data, they found than state CON for HHAs was associated with substantially lower rates of home health service utilization, but found no statistically significant association between home health $\mathrm{CON}$ and rehospitalization among those receiving home health care following discharge from a hospital stay (an indirect measure service quality). However, no published studies have examined the impact of HHA entry restrictions on direct measures of HHA-level quality. Our paper fills a gap in the literature by focusing on the association between home health $\mathrm{CON}$ and direct categorical measures of service quality at the home health agency level.

\section{Model}

\subsection{Data}

The home health agency quality ratings used in our analysis are from the Centers for Medicare and Medicaid Services (CMS) "Home Health Compare" database. CMS uses nine performance metrics to calculate quarterly "Star" ratings for each home health agency. The nine metrics include three measures of process quality: (1) timely initiation of care; (2) drug education on all medications provided to patient/caregiver; and (3) influenza immunization received for current flu season, and six outcome measures: (1) improvement in ambulation; (2) improvement in bed transferring; (3) improvement in bathing; (4) improvement in pain interfering with activity; (5) improvement in dyspnea; and (6) acute care hospitalization.

The methodology used by CMS to generate the "Star" ratings is described in detail on the CMS website (CMS 2015). In brief, each HHA's star rating is based on the agency's decile rank nationally for each of the nine measures (i.e., agencies in the top 10 percent for a specific measure are assigned a score of 5 , while those in the bottom 10 percent are assigned a score of 0.5 for that performance measure). If a particular 
HHA's performance measure value is not statistically different from the threshold value for the next highest decile, the HHA's score for that performance measure is adjusted to the next highest score. The adjusted ratings are averaged across the nine measures and rounded to the nearest half star $(5,4.5$, 4, etc.). Because most HHAs are not consistently in the top or bottom decile for all 9 measures, the majority of HHAs have an overall star rating between 2 and 4 stars.

We retrieved data for 22 agency-level quality measures (including the 9 measures used for Star ratings) from the CMS Home Health Compare database archive (https:// data.medicare.gov/data/archives/home-health-compare) by quarter from the last quarter of 2010 through the last quarter of 2013. We excluded all HHAs located in Alaska, Hawaii and the District of Columbia from the study sample. CMS Star ratings were not available for all years in our database, so we calculated an analog to the Star rating (labeled as "Star9") using the same method to assign scores to each agency in each quarter based on each HHA's decile rank. For consistency, the national decile rank thresholds for each performance metric were based on the distribution of the performance metric for all HHAs in all quarters in the database. Because the sample sizes used to generate agency-level performance measures are not reported, we were not able to make the adjustment for lack of statistical differences in decile ranks as employed in the CMS Star rating. However, among HHAs with both ratings, the Star9 rating is highly correlated with the CMS Star rating. Using an analogous method, we also created an alternative composite quality measure based on all 22 performance metrics in the CMS Home Health Compare database (labeled "Star22"). This alternative measure will be used to assess the robustness of our results to differences in the measurement of quality.

There were a total of 149,235 HHAs with quality scores in our initial sample, representing 13,133 unique HHAs across all quarters. Of those, 104,636 observations representing 10,095 unique HHAs had no missing data for the 9 performance metrics. The reports also include a code indicating whether the agency is investor-owned (forprofit), a government agency, or a private not-for-profit agency. We assigned a county code to each HHA based on the agency's reported zip code location using the countyzipcode crosswalk file for the fourth quarter of 2013 maintained by the U.S. Department of Housing and Urban Development (HUD 2015). However, some zip codes cover more than one county, so we created two alternative data sets, with the county code set to either the first or last county code for zip codes with multiple county codes.

Based on this county code, selected county-level area characteristics were obtained from the "Area Health Resources File" (http://ahrf.hrsa.gov/) and merged into the quality rating data based on county and calendar year for the quality data. Data for the prevalence of obesity $(\mathrm{BMI}>30)$ by state and year, obtained from various annual issues of "State of Obesity" reports (http://stateofobesity.org/files/stateofobesity 2014. pdf) produced by the Trust for America's Health (2014) were merged into the quality file based on the state and year for each agency in the sample.

Finally, measures of state-level barriers to entry for HHAs were merged into the quality data, as were measures of entry barriers for nursing homes and assisted living facilities (as potential substitutes for some forms of home health services). The two main forms of entry barriers are CON and "moratoria" on new entry. Even though a "moratorium" on new entry in practice is not a true moratorium (entry may occur), we treat it as a potentially more stringent barrier to entry than $\mathrm{CON}$ alone, given that every 
state with an entry moratorium for a provider type considered in this study also had $\mathrm{CON}$ in place for that provider type. Data for state-level entry barriers were obtained from National Council of State Legislators' website (http://www.ncsl.org/research/ health/con-certificate-of-need-state-laws.aspx\#Program). There was no change in any state regulation relating to entry barriers over the study period.

\subsection{Analytic approach}

Our primary measure of home health agency quality is the Star9 rating, which ranges from 0.5 to 5 on 0.5 increments. However, it would not be appropriate to consider this as a quasi-continuous outcome measure to be used as a dependent variable in a standard linear regression. Past literature suggests that simple quality measures that help differentiate "best" and "worst" health care providers are most salient to those selecting health care providers (Philips et al. 2007). Accordingly, we converted the Star9 rating into a three-level ordinal variable: "Low" quality (Star9 $\leq 2)$; "Medium" quality $(2<$ Star9 < 4); and "High" quality (Star9 $\geq 4)$. We used ordered logit regression to assess the association between state entry restrictions and HHA quality level, adjusted for other factors potentially affecting quality. Specifically:

Quality $=f$ (Entry Regulation, Urban/Rural, Population Characteristics, Quarter, Year)

Unfortunately, as noted, there was no change in any state regulation relating to entry barriers over the study period. Thus, using the popular "differences in differences" analytic approach (Dimick and Ryan 2014) is not possible in this case. The analytic model is essentially a cross-sectional comparison using multiple quarters of cross-sectional data with repeated quality measures for HHAs, using covariate adjustment for HHA and county-level factors potentially affecting quality. Standard errors for model coefficients were estimated accounting for repeated quality measures for specific HHAs over time.

The state entry barrier for HHAs was defined as a binary variable equal to 1 for the 17 states where either $\mathrm{CON}$ or a moratorium for HHAs (HHA-CON or CON \& Moratorium) was present. Only one state (Mississippi) had both CON and a moratorium restricting HHA entry. For nursing home entry, we distinguished between the 34 states in the study sample with nursing home CON (Nursing Home-CON) from the subset of 15 states which also had a nursing home moratorium in place (Nursing Home-CON \& Moratorium). Finally, we accounted for the presence of CON for assisted living facilities (Assisted Living-CON) in 5 states (no states had a moratorium for assisted living). During the study period, all of the states with CON for HHAs also had CON for nursing homes, and all of the states with CON for assisted living facilities also had CON for HHAs. Therefore, the analysis focused on the potential for other forms of entry restrictions to either reinforce or counteract the impact of HHA restrictions.

Agency ownership was defined by a binary variable equal to 1 if the agency was for-profit (Investor-Owned), or a private voluntary agency (Private Not-for-Profit), with government agencies as the reference category. Some past studies of nursing 
home quality suggest service quality among for-profit providers is lower than among not-for-profit providers (Grabowski and Hirth 2003). Urban/rural status was measured as a set of binary variables based on the county-level Rural-Urban Continuum Code (RUCC) in each year: "Metro-Large" for counties classified as part of a metro area with a population greater than 250,000 (i.e., counties with RUCC of 1 or 2); "MetroSmall" (counties with an RUCC of 3, 4 or 5); "Rural-Adjacent to Metro" for rural counties adjacent to a metro county (RUCC of 6 or 8); and "Rural" (RUCC of 7 or 9) as the reference category.

The model also accounts for county-area population variables; specifically real per capita income, the percent of the population with household income below the Federal poverty level, the percent of the population age 65 or higher, and the unemployment rate. These variables may capture some variation in payer mix across HHAs. The model also includes a measure of the prevalence of obesity in a state's population (county level measures of obesity rates were not available). It might be more difficult for agencies with more obese patients to attain quality metrics given their greater resource requirements, compared to agencies with fewer obese patients. Finally, the model included binary variables for each quarter and year in the data, with the last quarter of 2010 being the reference category. Descriptive statistics for all study variables are reported in Table 1.

We specified four main models with ordinal quality as the dependent variable. The primary measure of ordinal quality used was based on Star9 quality categories (Low, Medium, and High). The first model included only the HHA entry restriction variable and the year-quarter dummy variables as independent variables. The second model added variables for entry restrictions for the remaining service types, and the third model added agency ownership and urban/rural location variables. The final model added county-level socioeconomic variables. All models were estimated using Stata Version 14 (www.stata.com), with standard error estimates accounting for clustering across repeated quality measures for specific HHAs over time.

To assess the robustness of our quality model results, we constructed an alternative grouping of the Star9 score values based on approximate tertiles of Star9 score distribution: Tertile 1 (Star9 < 2.5; 26.8\%); Tertile $2(2.5 \leq$ Star9 < 3.0; 36.1\% 0: and Tertile 3 (Star9 $\geq 3.0 ; 37.1 \%$ ). We also estimated quality models using ordered categories of Star22 score values. Finally, both the Star9 and Star22 scores (i.e., number of stars) were used as quasi-continuous quality outcome measures in a doubly truncated linear regression model.

\section{Results}

Descriptive statistics for model variables in the study sample are reported in Table 1. Overall, $23.7 \%$ of the HHAs in the sample were rated in the "High" quality category. However, $26.1 \%$ of HHAs in states without CON for HHAs were rated in the High quality category, compared to $10.8 \%$ of HHAs in states with CON for HHAs. Thus, HHAs in CON states were about $58 \%$ less likely to be rated as High quality $(p<.01)$. HHAs in CON states also were about $30 \%$ more likely to be rated as "Medium" quality compared to HHAs in states without CON for HHAs. In contrast, there was 
Table 1 HHA sample mean values for quality model variables, by state HHA CON status. Source: see text

\begin{tabular}{|c|c|c|c|}
\hline & $\begin{array}{l}\text { Full sample } \\
(\mathrm{N}=147,986)\end{array}$ & $\begin{array}{l}\text { HHA CON } \\
(\mathrm{N}=23,202)\end{array}$ & $\begin{array}{l}\text { No HHA CON } \\
(\mathrm{N}=124,784)\end{array}$ \\
\hline \multicolumn{4}{|l|}{ Star9 quality category } \\
\hline Low $(\leq 2)$ & 0.268 & 0.278 & 0.267 \\
\hline Medium $(>2$ but $<4$ ) & 0.494 & 0.614 & 0.472 \\
\hline $\operatorname{High}(\geq 4)$ & 0.237 & 0.108 & 0.261 \\
\hline \multicolumn{4}{|l|}{ State entry regulations } \\
\hline $\mathrm{HHA}-\mathrm{CON}$ or $\mathrm{CON} \&$ Moratorium & 0.157 & 1 & 0 \\
\hline Nursing Home-CON & 0.572 & 1 & 0.502 \\
\hline Nursing Home-CON \& Moratorium & 0.245 & 0.387 & 0.218 \\
\hline Assisted Living—CON & 0.066 & 0.415 & 0 \\
\hline \multicolumn{4}{|l|}{ Agency ownership/location } \\
\hline Investor Owned & 0.779 & 0.543 & 0.823 \\
\hline Private Not-for-Profit & 0.155 & 0.273 & 0.133 \\
\hline Government/Other & 0.066 & 0.183 & 0.044 \\
\hline Metro-Large & 0.590 & 0.294 & 0.644 \\
\hline Metro-Small & 0.239 & 0.348 & 0.219 \\
\hline Rural-Adjacent to Metro & 0.099 & 0.196 & 0.081 \\
\hline Rural-Not Adjacent & 0.072 & 0.162 & 0.056 \\
\hline \multicolumn{4}{|l|}{ County-area characteristics } \\
\hline Real Per Capita Income $(\$ 000)$ & 43.80 & 40.23 & 44.46 \\
\hline Population $<$ Poverty level $(\%)$ & 17.08 & 18.61 & 16.79 \\
\hline Unemployment Rate (\%) & 4.68 & 4.57 & 4.70 \\
\hline Population Age $65+(\%)$ & 13.64 & 15.80 & 13.24 \\
\hline Obesity (State Population, \%) & 28.60 & 30.33 & 28.28 \\
\hline
\end{tabular}

little difference in the sample proportions of HHAs rated as "Low" quality across HHA CON status. Of course, these simple frequency comparisons are not adjusted for other factors potentially affecting quality.

One such factor is agency ownership categories. HHAs in CON states were less likely to be investor-owned, and more likely to be private not-for-profit, than HHAs in states without $\mathrm{CON}$ for HHAs. In terms of urban/rural location, HHAs in states with CON for HHAs generally were located in more rural areas compared to other HHAs in the sample. County-area socioeconomic characteristics for HHAs also difference by CON status: HHAs in CON states were located in counties with lower per capita income, higher poverty rates, a higher percentage of county population aged 65 years or older, and in states with greater obesity, compared to other HHAs in the sample. Differences in the area unemployment rate were comparatively small.

Results for the multivariable ordered logistic regression model using the Star9 quality categories are reported in Table 2 . When the quality model only includes a variable indicating the presence of $\mathrm{CON}$ (or a moratorium) for HHAs, along with 
Table 2 Ordered logit models for "Star9" quality categories for home health agencies, quarterly data for Q4-2010 to Q4-2013. Source: see text

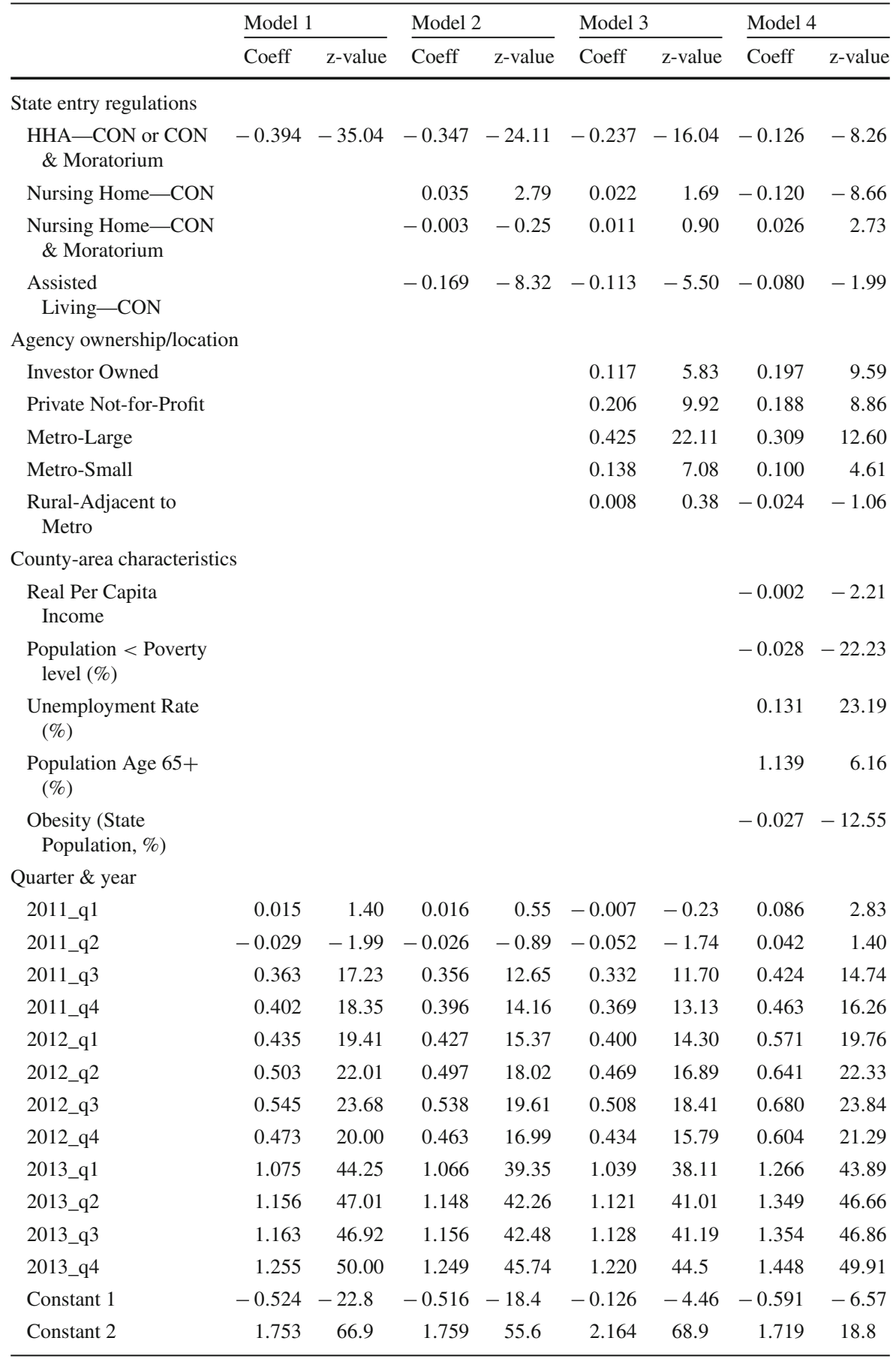


quarter-year dummy variables (Model 1), the coefficient of the HHA entry restriction variable indicates that HHAs in states with HHA entry restrictions were likely to have a lower quality rating category, compared to HHAs in other states $(p<.001)$. However, as noted, all of the states with CON for HHAs also had CON for nursing homes, so the group of "comparison" states in this instance consists of a mixture of 14 states with no $\mathrm{CON}$ at all and 34 states with nursing home CON (plus moratoria in 15 of those 34 states) but no CON for HHAs.

Model 2 adds measures for state entry restrictions for related provider categories. The coefficient of the indicator for nursing home CON (with or without a moratorium) is positive but small though statistically significant $(p<.05)$, whereas the estimated coefficient of the variable indicating the presence of a nursing home moratorium (in addition to CON) was not statistically significant. However, the coefficient for the indicator for assisted living CON indicates HHAs located in states with this entry restriction are more likely to have a lower HHA quality rating category $(p<.01)$, compared to HHAs in states without $\mathrm{CON}$ for assisted living.

Adding variables for HHA ownership type and urban-rural location (Model 3) reduces the magnitude of the estimated coefficients for the HHA and assisted living $\mathrm{CON}$ indicators, but both remain statistically significant $(p<.01)$. For-profit or private not-for-profit ownership status is associated with a higher quality rating category compared to government agencies $(p<.01)$, and a metro-area location (large or small) is associated with a higher quality rating category compared to rural locations $(p<.01)$.

Finally, when county-area population variables are added to the model (Model 4), the magnitude of the estimated coefficient for the HHA and assisted living CON indicators are smaller than in the other model specifications but they remain statistically significant $(p<.05)$. In this model specification, the coefficient of the indicator variables for nursing home $\mathrm{CON}$ (with or without a moratorium) is negative and statistically significant $(p<.01)$, whereas the estimated coefficient of the variable indicating the presence of a nursing home moratorium in addition to $\mathrm{CON}$ was positive but small though statistically significant $(p<.05)$. Thus, HHAs in states with nursing home CON are likely to have lower quality rating categories compared to states with no $\mathrm{CON}$, but the addition of a nursing home moratorium to nursing home $\mathrm{CON}$ is not associated with lower HHA quality.

A potential limitation of the standard ordered logistic regression model is that model estimates are obtained by imposing a proportionality of odds constraint across response categories. An approximate likelihood-ratio test applied to Model 4 indicated the null hypothesis of proportional odds across response categories was rejected $\left(\chi^{2}=9,502\right.$; $p<.001)$. Thus, we estimated a generalized ordered logistic regression using the same variables used in Model 4. The results, shown in Table 3, confirm that the coefficients of the state entry regulation variables substantially differ across response levels.

However, given the overlapping nature of state entry restrictions across types of providers, in general it is difficult to discern the overall impact of different combinations of entry restrictions for various service providers on quality levels simply by examining the estimated coefficients of each category of regulation in isolation. To address this issue, we used the estimated coefficients in Table 3 to generate predicted quality levels for different combinations of entry restrictions. Figure 1 illustrates the 
Table 3 Generalized ordered logit models for "Star9" quality categories for home health agencies, quarterly data for Q4-2010 to Q4-2013. Source: see text. Level 3 (High Quality) is the reference category.

\begin{tabular}{|c|c|c|c|c|}
\hline & \multicolumn{2}{|l|}{ Level 1} & \multicolumn{2}{|l|}{ Level 2} \\
\hline & Coeff & z-value & Coeff & z-value \\
\hline \multicolumn{5}{|l|}{ State entry regulations } \\
\hline HHA-CON or CON \& Moratorium & 0.197 & 8.16 & -0.755 & -24.82 \\
\hline Nursing Home $-\mathrm{CON}$ & -0.032 & -1.86 & -0.252 & -14.17 \\
\hline Nursing Home-CON \& Moratorium & 0.034 & 1.89 & 0.099 & 4.99 \\
\hline Assisted Living—CON & -0.099 & -3.17 & 0.102 & 2.31 \\
\hline \multicolumn{5}{|l|}{ Agency ownership/location } \\
\hline Investor Owned & 0.208 & 7.77 & 0.267 & 7.79 \\
\hline Private Not-for-Profit & 0.513 & 17.25 & -0.067 & -1.80 \\
\hline Metro-Large & 0.259 & 7.82 & 0.427 & 11.50 \\
\hline Metro-Small & 0.254 & 8.64 & 0.030 & 0.86 \\
\hline Rural-Adjacent to Metro & 0.083 & 2.71 & -0.208 & -5.34 \\
\hline \multicolumn{5}{|l|}{ County-area characteristics } \\
\hline Real Per Capita Income & -0.006 & -6.76 & 0.004 & 4.82 \\
\hline Population < Poverty level (\%) & -0.046 & -29.69 & -0.005 & -3.18 \\
\hline Unemployment Rate (\%) & 0.195 & 25.25 & 0.054 & 6.97 \\
\hline Population Age 65+ (\%) & 3.711 & 14.19 & -0.868 & -3.19 \\
\hline Obesity (State Population, \%) & -0.061 & -21.07 & 0.012 & 4.11 \\
\hline \multicolumn{5}{|l|}{ Quarter \& year } \\
\hline 2011_q1 & 0.105 & 3.61 & 0.015 & 0.44 \\
\hline 2011_q2 & 0.094 & 3.22 & -0.089 & -2.52 \\
\hline 2011_q3 & 0.600 & 20.10 & 0.046 & 1.30 \\
\hline 2011_q4 & 0.664 & 22.20 & 0.059 & 1.70 \\
\hline 2012_q1 & 0.822 & 26.64 & 0.105 & 2.95 \\
\hline 2012_q2 & 0.923 & 29.71 & 0.143 & 4.04 \\
\hline 2012_q3 & 0.996 & 31.87 & 0.157 & 4.41 \\
\hline 2012_q4 & 0.946 & 30.47 & 0.045 & 1.25 \\
\hline 2013_q1 & 1.701 & 49.62 & 0.719 & 21.12 \\
\hline 2013_q2 & 1.784 & 51.49 & 0.816 & 24.11 \\
\hline 2013_q3 & 1.770 & 51.43 & 0.832 & 24.69 \\
\hline 2013_q4 & 1.855 & 53.15 & 0.940 & 28.03 \\
\hline Constant & 1.037 & 8.67 & -2.361 & -18.80 \\
\hline
\end{tabular}

predicted impact of increasingly all-encompassing state entry restrictions on a home health agency's likelihood of attaining a specific quality rating category. The first column of Fig. 1 reports the estimated probability of attaining Low, Medium, or High levels for the Star9 ordinal quality measure for HHAs in states no entry restrictions for HHAs, nursing homes, or assisted living facilities ("None"). For HHAs in states with no entry restrictions, the results indicated predicted probabilities of $0.271,0.456$, 


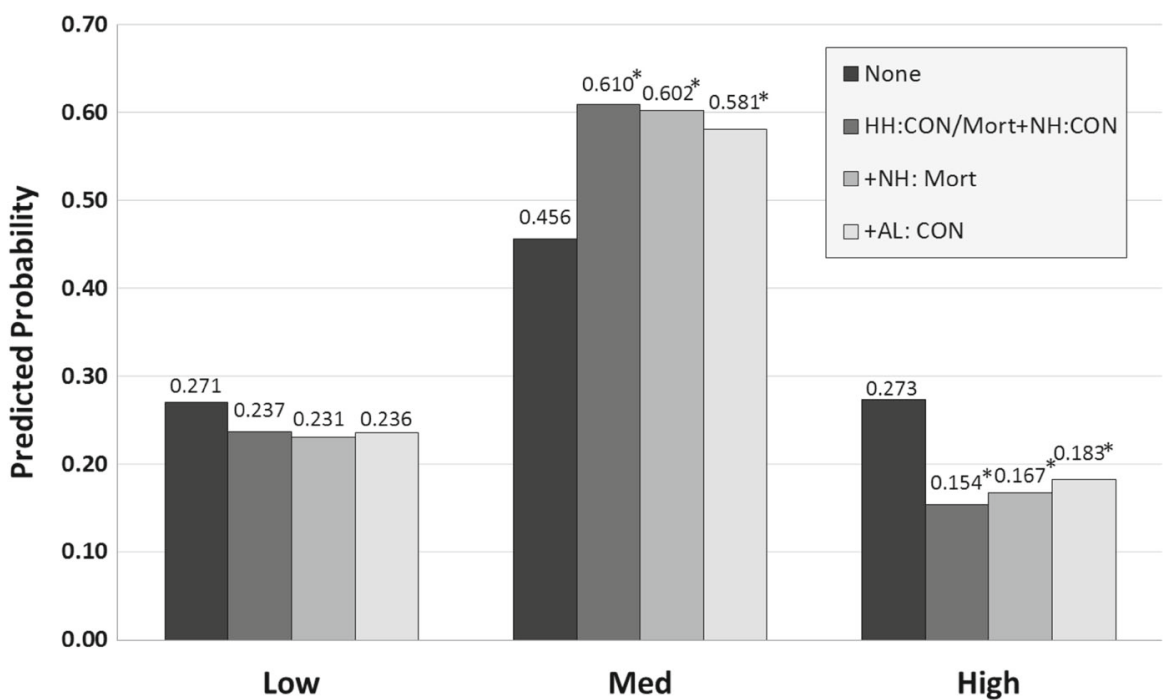

Fig. 1 Predicted probabilities from generalized ordered logit model (Table 3) for Home Health Star9 quality rating categories, by state entry restrictions for home health and related service providers. $H H$ home health agency, $\mathrm{NH}$ nursing home, $A L$ assisted living facility, $C O N$ certificate-of-need, Mort moratorium. *Difference in predicted probability compared to "None" is statistically significant $(p<.01)$

and 0.273 , for Low, Medium and High quality categories, respectively, projected over the entire sample.

For HHAs located in states with CON for both HHAs and nursing homes (but no nursing home moratorium), the estimated probabilities for quality levels were $0.237,0.610$, and 0.154 for Low, Medium and High quality categories, respectively. These estimated values for the predicted probabilities were statistically different ( $p<$ .01) from the corresponding probability estimates for HHAs in states with no entry restrictions for the Medium and High quality categories, but not the Low category. Accounting for the presence of state moratoria on new nursing home capacity (in addition to CON) had little impact on the predicted likelihood of a Low, Medium or High HHA quality rating category, compared to just nursing home CON. Finally, adding the impact of state entry restrictions for assisted living to other entry restrictions slightly reduced the point estimate of the likelihood of a Medium HHA quality rating category, and slightly increased the point estimate of the likelihood of a High quality rating category, compared to HHAs in states CON states without CON for assisted living. However, these differences are not statistically significant. HHAs in these most restricted states had estimated predicted probabilities of $0.236,0.581$ and 0.183 for Low, Medium and High quality categories, respectively.

Thus, compared to HHAs in states with HHA CON and nursing home CON, HHAs in states with no entry restrictions were $25 \%$ less likely to have a Medium quality rating and $76 \%$ more likely to have a High quality rating. Similarly, compared HHAs in the most restrictive states, HHAs in states with no entry restrictions were $22 \%$ less likely to have a Medium quality rating, and $49 \%$ more likely to have a High quality rating. 
As a contrast, we estimated the same probabilities using the results of Model 4 (Table 2), which imposed a proportional odds restriction on model coefficients. As shown in Fig. 2, the estimated probabilities of Low and High quality are greater than the associated probabilities using the generalized model results. The estimated probabilities for Medium quality are lower for HHAs in states with CON, but slightly lower for HHAs in states without CON. Thus, while more restrictive entry regulation remains associated with lower HHA quality rating categories in the standard ordered logit model, the magnitude of the differences are muted by the proportional odds restriction. For example, in Fig. 2, compared to HHAs in the most restrictive states, HHAs in states with no entry restrictions were $20 \%$ less likely to have a Low quality rating category and $32 \%$ more likely to have a High quality rating category, but differences in the likelihood of a Medium quality rating were small.

\section{Discussion}

Most of the existing literature evaluating state CON programs has focused on the cost impact of hospital CON. The strong consensus of this literature is that hospital CON is associated with similar or higher inpatient costs (Conover and Sloan 1998; Antel et al. 1995; Ohlhausen 2015). Less attention has been directed toward the impact of hospital CON on hospital quality, with some studies suggesting improved quality for complex inpatient procedures (Vaughan-Sarrazin et al. 2002), but lower quality for more routine hospital care (Stratmann and Wille 2016). Studies examining nursing home CON conclude CON does not reduce, and may increase, nursing home expenditures (Grabowski et al. 2003; Rahman et al. 2016), and may be associated with lower quality (Ching et al. 2015). Comparatively few studies have examined CON for HHAs. Rahman et al. (2016), using state-level expenditure data, found that HHA CON tends to shift expenditures from HHAs to nursing homes, and Polsky et al. (2014), using Medicare claims data, found no statistically significant association between state $\mathrm{CON}$ for HHAs and risk of rehospitalization following receipt of post-acute care home health services (an indirect measure home health service quality).

Our results, using direct measures of quality of care for HHAs, suggest that HHAs located in states with CON for HHAs were less likely to have High quality ratings compared to HHAs in states without $\mathrm{CON}$ for HHA. A plausible causal pathway for adverse quality effect is the potential impact of entry restrictions on the degree of structural competition among HHAs in states with CON for HHAs. We have no direct measures of structural competition in states with and without CON for HHAs. However, Polsky et al. (2014) estimate that states with CON for HHAs have about half as many HHAs per 100,000 Medicare recipients compared to states without CON for HHAs, resulting in an average Herfindahl-Hirschman Index (HHI) of 3256 in CON states compared to 2259 in non-CON states (p. 2). Huang and Hirth (2016), examining the impact of the Five-Star rating reports for nursing homes, found that consumers were more responsive to quality information in less concentrated markets. While their study focuses on pricing practices, it is plausible to suggest less competitive pressure to lower prices also translates into less competitive pressure to maintain or improve service 
quality. Thus, the higher HHA quality ratings in states without $\mathrm{CON}$ for HHAs, relative to those with CON, is most likely a result of enhanced competition among HHAs.

Ordered logit model results using tertile Star9 categories, reported in Table 4, were substantively similar in terms of the signs and statistical significance of the estimated coefficients for the entry restriction variables in the ordered logit model using Low, Medium and High Star9 categories (Table 2), though the estimated impact of entry restriction on HHA quality using the tertile Star9 categories was slightly smaller compared to results using the Low, Medium and High Star9 categories. This may reflect greater of salience of quality extremes to HHA consumers compared to tertile levels. Ordered logit models using Low, Medium and High Star22 categories produced results similar to the model results for the tertile Star9 categories. CMS selected the nine specific metrics to incorporate into their Five-Star rating system based on their assessment that these were the most reliable and salient quality indicators among the 22 indicators. Including the other 13 measures with equal weighting may increase the level of "noise" in Star22 as a signal of HHA quality, which would attenuate the estimated impact of entry restrictions on HHA quality levels measured by Star22.

We also estimated doubly truncated regression models using STAR9 and STAR22 scores as a quasi-continuous variable, with limit values at one-half and five stars. As reported in Table 5, the estimated coefficient of the HHA entry restriction variable is negative but not statistically significant for models using either quality metric. Recall that about half of the HHAs in the sample are in the "Medium" quality category. Computationally, regression determines model parameters which fit the model well primarily where the data are most “dense." Regression model parameters using STAR9 or STAR22 as a continuous dependent variable thus mainly reflect the impact of entry restrictions on variations in HHA quality scores within the medium quality category. The attenuated estimated impact of entry restrictions on HHA quality in the truncated regression model could reflect a limited impact of entry restrictions on variations across HHAs quality within the medium category, compared to the more extreme impact on the highest categories indicated in the generalized ordered logit model. In other words, the "star" scores are inherently ordinal, and the assumption of linearity in the relationship between half-star differences in indicators of quality and the extent of state entry restrictions is inconsistent with literature suggesting that quality extremes are more likely to affect provider selection.

\subsection{Limitations}

The most significant limitation of this study is the lack of variation over time in entry restrictions within states over the study period. Although the quality models adjust for county-level population characteristics potentially impacting HHA quality, it is possible that other factors at the county or state level not accounted for in our model have an impact on HHA quality. If these unobserved factors have a negative impact on quality and are correlated with presence of state entry restrictions, the estimated impact of entry restrictions on HHA quality could be substantially overstated or even entirely spurious. 
Another limitation relates to the use of the HHA's reported zipcode to define countyarea characteristics for the HHA. The reported zipcode is associated with the location of the principal administrative office for the HHA. The HHA's service area is likely to cover multiple zipcodes, which in turn may cover multiple counties. Moreover, even if the assignment of a specific county to an HHA correctly approximates the HHA's service area, the county-level population characteristics may not accurately reflect the characteristics of the specific patient population served by an HHA.

Finally, the impact of entry restrictions on HHA quality operates as a "black box" in our quality analysis. We have no direct measures of variation in the degree to which entry restrictions are binding across states with entry restrictions, nor do we have any direct measures of the resulting impact on market-level structural concentration. It may be plausible to suggest that the observed relationship between greater entry restrictions and lower HHA quality most likely results from less competition among HHAs in entry-restricted states, but ultimately that is a conjecture based on indirect evidence.

\section{Conclusion}

No previous research assessing the impact of CON programs on quality of care has focused on the impact of CON for HHAs using direct measures of HHA quality. Our study assessed the impact of state CON programs for HHAs on HHA quality ratings. The results indicated that HHAs in states with CON for HHAs, and CON for potential substitute service providers, were likely to receive lower quality ratings compared to HHAs in states without CON, adjusted for HHA ownership type and area population characteristics. Specifically, compared HHAs in the states with the most comprehensive entry restrictions, HHAs in states with no entry restrictions were $22 \%$ less likely to have a Medium quality rating, and $49 \%$ more likely to have a High quality rating. A plausible causal pathway for an adverse effect of $\mathrm{CON}$ on quality is reduced structural competition among HHAs due to entry restrictions in states with CON for HHAs. However, our data do not provide any direct measures of structural competition in states with and without CON for HHAs. Further study is needed to assess reasons for the observed adverse impact of state entry restrictions on HHA quality.

Acknowledgements Partial funding support was provided by the Arkansas Center for Research in Economics.

\section{Compliance with ethical standards}

Conflicts of interest All the authors declare that they have no conflict of interest.

Open Access This article is distributed under the terms of the Creative Commons Attribution 4.0 International License (http://creativecommons.org/licenses/by/4.0/), which permits unrestricted use, distribution, and reproduction in any medium, provided you give appropriate credit to the original author(s) and the source, provide a link to the Creative Commons license, and indicate if changes were made.

\section{Appendix}

See Tables 4, 5 and Fig 2. 
Table 4 Ordered logit models for "Star9" tertile categories for home health agencies, quarterly data for Q4-2010 to Q4-2013. Source: see text

\begin{tabular}{|c|c|c|c|c|c|c|c|c|}
\hline & \multicolumn{2}{|c|}{ Model 1} & \multicolumn{2}{|c|}{ Model 2} & \multicolumn{2}{|c|}{ Model 3} & \multicolumn{2}{|c|}{ Model 4} \\
\hline & Coeff & z-value & Coeff & z-value & Coeff & z-value & Coeff & z-value \\
\hline \multicolumn{9}{|l|}{ State entry regulations } \\
\hline $\begin{array}{l}\mathrm{HHA}-\mathrm{CON} \text { or } \mathrm{CON} \& \\
\text { Moratorium }\end{array}$ & -0.341 & -29.51 & -0.312 & -20.78 & -0.226 & -14.67 & -0.101 & -6.32 \\
\hline Nursing Home $-\mathrm{CON}$ & & & 0.094 & 7.56 & 0.078 & 6.25 & -0.088 & -6.46 \\
\hline $\begin{array}{l}\text { Nursing Home-CON } \\
\& \text { Moratorium }\end{array}$ & & & 0.030 & 2.43 & 0.047 & 3.73 & 0.062 & 4.62 \\
\hline Assisted Living—CON & & & -0.211 & -10.04 & -0.153 & -7.23 & -0.045 & -2.08 \\
\hline \multicolumn{9}{|l|}{ Agency ownership/location } \\
\hline Investor Owned & & & & & 0.119 & 5.83 & 0.208 & 10.04 \\
\hline Private Not-for-Profit & & & & & 0.297 & 9.92 & 0.275 & 12.71 \\
\hline Metro-Large & & & & & 0.404 & 22.11 & 0.313 & 12.51 \\
\hline Metro-Small & & & & & 0.150 & 7.08 & 0.129 & 5.87 \\
\hline $\begin{array}{l}\text { Rural-Adjacent to } \\
\text { Metro }\end{array}$ & & & & & 0.050 & 0.38 & 0.023 & 1.00 \\
\hline \multicolumn{9}{|l|}{ County-area characteristics } \\
\hline Real Per Capita Income & & & & & & & -0.002 & -2.64 \\
\hline $\begin{array}{l}\text { Population }<\text { Poverty } \\
\text { level }(\%)\end{array}$ & & & & & & & -0.029 & -23.55 \\
\hline $\begin{array}{l}\text { Unemployment Rate } \\
(\%)\end{array}$ & & & & & & & 0.144 & 25.06 \\
\hline Population Age $65+(\%)$ & & & & & & & 1.828 & 9.78 \\
\hline $\begin{array}{l}\text { Obesity (State } \\
\text { Population, \%) }\end{array}$ & & & & & & & -0.034 & -15.65 \\
\hline \multicolumn{9}{|l|}{ Quarter \& year } \\
\hline 2011_q1 & 0.011 & 0.42 & 0.013 & 0.48 & -0.007 & -0.23 & 0.078 & 2.75 \\
\hline 2011_q2 & -0.019 & -0.70 & -0.016 & -0.60 & -0.052 & -1.74 & 0.048 & 1.70 \\
\hline 2011_q3 & 0.380 & 14.58 & 0.375 & 14.29 & 0.332 & 11.7 & 0.443 & 16.45 \\
\hline 2011_q4 & 0.441 & 16.93 & 0.437 & 16.68 & 0.369 & 13.13 & 0.505 & 18.81 \\
\hline 2012_q1 & 0.477 & 18.39 & 0.473 & 18.11 & 0.400 & 14.3 & 0.623 & 22.77 \\
\hline 2012_q2 & 0.573 & 22.07 & 0.570 & 21.81 & 0.469 & 16.89 & 0.721 & 26.37 \\
\hline 2012_q3 & 0.634 & 24.44 & 0.629 & 24.08 & 0.508 & 18.41 & 0.780 & 28.54 \\
\hline 2012_q4 & 0.581 & 22.38 & 0.575 & 21.99 & 0.434 & 15.79 & 0.723 & 26.49 \\
\hline 2013_q1 & 1.186 & 45.82 & 1.181 & 45.30 & 1.039 & 38.11 & 1.400 & 50.17 \\
\hline 2013_q2 & 1.263 & 48.55 & 1.259 & 48.09 & 1.121 & 41.01 & 1.479 & 52.83 \\
\hline 2013_q3 & 1.287 & 49.23 & 1.283 & 48.80 & 1.128 & 41.19 & 1.501 & 53.49 \\
\hline 2013_q4 & 1.375 & 52.41 & 1.372 & 51.96 & 1.220 & 44.5 & 1.592 & 56.47 \\
\hline Constant 1 & -0.459 & -23.1 & -0.413 & -19.75 & -0.024 & -0.83 & -0.534 & -5.85 \\
\hline Constant 2 & 1.160 & 57.6 & 1.206 & 56.92 & 1.604 & 53.73 & 1.114 & 12.20 \\
\hline
\end{tabular}


Table 5 Truncated linear regression model for "Star9" and "Star22" scores for home health agencies, quarterly data for Q4-2010 to Q4-2013 (Lower limit = 1/2 star; Upper limit = 5 stars). Source: see text

\begin{tabular}{|c|c|c|c|c|}
\hline & \multicolumn{2}{|c|}{ Star9 Score } & \multicolumn{2}{|c|}{ Star22 Score } \\
\hline & Coeff & z-value & Coeff & z-value \\
\hline \multicolumn{5}{|l|}{ State entry regulations } \\
\hline HHA-CON or CON \& Moratorium & 0.040 & 1.13 & -0.021 & -1.05 \\
\hline Nursing Home $-\mathrm{CON}$ & -0.011 & -0.61 & -0.084 & -5.06 \\
\hline Nursing Home $-\mathrm{CON} \&$ Moratorium & 0.050 & 1.70 & 0.044 & 2.27 \\
\hline Assisted Living-CON & -0.014 & -0.44 & -0.002 & -0.27 \\
\hline \multicolumn{5}{|l|}{ Agency ownership/location } \\
\hline Investor Owned & 0.083 & 2.91 & 0.055 & 2.35 \\
\hline Private Not-for-Profit & 0.185 & 6.22 & 0.076 & 3.04 \\
\hline Metro-Large & 0.096 & 2.85 & -0.010 & -0.34 \\
\hline Metro-Small & 0.071 & 2.40 & -0.048 & -1.91 \\
\hline Rural-Adjacent to Metro & 0.040 & 1.29 & -0.042 & -1.61 \\
\hline \multicolumn{5}{|l|}{ County-area characteristics } \\
\hline Real Per Capita Income & -0.003 & -3.59 & -0.003 & -3.34 \\
\hline Population < Poverty level (\%) & -0.018 & -11.3 & -0.012 & -9.34 \\
\hline Unemployment Rate (\%) & 0.095 & 12.59 & 0.088 & 12.81 \\
\hline Population Age 65+ (\%) & 1.548 & 6.19 & 0.724 & 3.33 \\
\hline Obesity (State Population, \%) & -0.031 & -11.11 & -0.008 & -3.44 \\
\hline \multicolumn{5}{|l|}{ Quarter \& year } \\
\hline 2011_q1 & 0.047 & 7.15 & 0.071 & 11.41 \\
\hline 2011_q2 & 0.053 & 7.04 & 0.098 & 13.89 \\
\hline 2011_q3 & 0.305 & 30.28 & 0.322 & 39.06 \\
\hline 2011_q4 & 0.349 & 33.42 & 0.407 & 47.15 \\
\hline 2012_q1 & 0.441 & 33.45 & 0.530 & 46.88 \\
\hline 2012_q2 & 0.486 & 36.42 & 0.594 & 51.83 \\
\hline 2012_q3 & 0.538 & 40.03 & 0.647 & 55.90 \\
\hline 2012_q4 & 0.605 & 42.40 & 0.702 & 60.14 \\
\hline 2013_q1 & 0.865 & 55.82 & 0.859 & 64.68 \\
\hline 2013_q2 & 0.913 & 58.91 & 0.898 & 67.20 \\
\hline 2013_q3 & 0.921 & 59.38 & 0.926 & 69.36 \\
\hline 2013_q4 & 0.966 & 61.96 & 0.962 & 71.51 \\
\hline Constant & 2.716 & 22.74 & 2.254 & 21.99 \\
\hline Sigma & 0.824 & 183.44 & 0.700 & 186.91 \\
\hline \#Truncated (at 0.5 or 5.0 ) & 2.805 & & 1.747 & \\
\hline
\end{tabular}




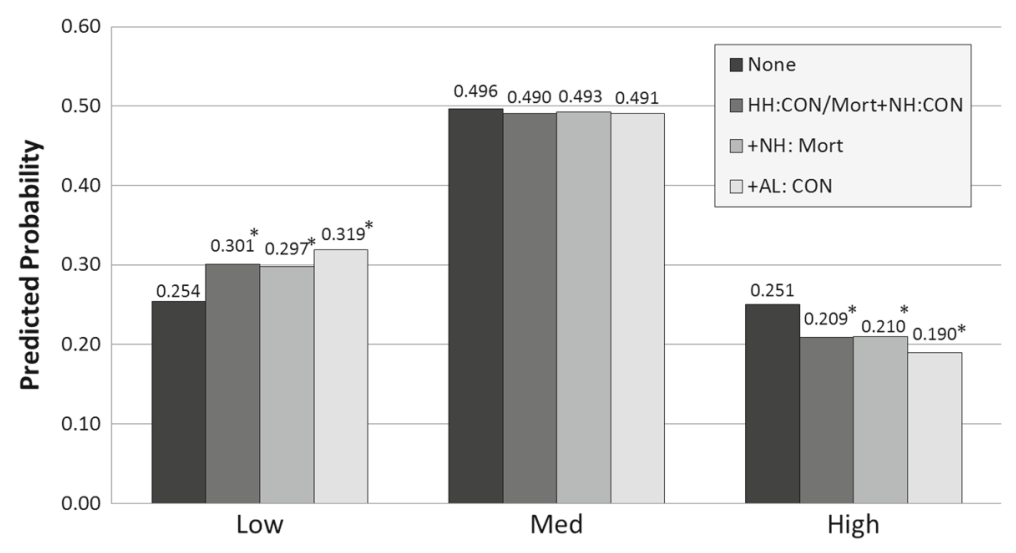

Fig. 2 Predicted probabilities from ordered logit model (Table 2, Model 4) for Home Health Star9 quality rating categories, by state entry restrictions for home health and related health service providers. $H H$ home health agency, $\mathrm{NH}$ nursing home, $A L$ assisted living facility, $C O N$ certificate-of-need, Mort moratorium. *Difference in predicted probability compared to "None" is statistically significant $(p<.01)$

\section{References}

Antel, J., Ohsfeldt, R., \& Becker, E. (1995). State regulation and hospital cost performance. Review of Economics and Statistics, 77(3), 416-422.

Bloom, N., Propper, C., Seiler, S., \& Van Reenen, J. (2015). The impact of competition on management quality: Evidence from public hospitals. Review of Economic Studies, 82(2), 457-489.

Centers for Medicare and Medicaid Services. (2015). Home health compare (HHC) star ratings methodology. March 16, https:/www.cms.gov/Medicare/Quality-Initiatives-Patient-Assessment-Instruments/ HomeHealthQualityInits/Downloads/Home-Health-Star-Ratings-Methodology-updated-3-16-15. pdf.

Centers for Medicare and Medicaid Services. (2017). National health expenditure data. https://www.cms.gov/Research-Statistics-Data-and-Systems/Statistics-Trends-and-Reports/ NationalHealthExpendData/index.html. Accessed 9 July 2017.

Ching, A., Hayashi, F., \& Wang, H. (2015). Quantifying the impacts of limited supply: The case of nursing homes. International Economic Review, 56(4), 1291-1322.

Conover, C., \& Sloan, F. (1998). Does removing certificate-of-need regulations lead to a surge in health care spending? Journal of Health Politics, Policy and Law, 23(3), 455-81.

David, G., \& Polsky, D. (2014). Economics of home health services. In A. J. Culyer (Ed.), Encyclopedia of health economics (Vol. 1, pp. 477-483). The Netherlands: Elsevier.

Dimick, J., \& Ryan, A. (2014). Methods for evaluating changes in health care policy: The difference-indifferences approach. JAMA, 312(22), 2401-2402.

Federal Trade Commission and U.S. Department of Justice. (2004). Improving health care: A dose of competition. https:/www.ftc.gov/sites/default/files/documents/reports/improving-health-care-dosecompetition-report-federal-trade-commission-and-department-justice/040723healthcarerpt.pdf. Accessed 7 May 2015.

Freeman, L. (1995). Home-sweet-home health care. Monthly Labor Review, 118(5), 3-11.

Gaynor, M., \& Vogt, W. (2000). Antitrust and competition in health care markets. In J. P. Newhouse (Ed.), Handbook of health economics. Amsterdam: Elsevier.

Grabowski, D., \& Hirth, R. (2003). Competitive spillovers across non-profit and for-profit nursing homes. Journal of Health Economics, 22(1), 1-22.

Grabowski, D., Ohsfeldt, R., \& Morrisey, M. (2003). The effects of CON repeal on medicaid nursing home and long term care expenditures. Inquiry, 40, 146-157.

Grabowski, D., \& Town, R. (2011). Does information matter? Competition, quality, and the impact of nursing home report cards. Health Services Research, 46(6, Part I), 1698-1719. 
Gutacker, N., Siciliani, L., Moscelli, G., \& Gravelle, H. (2016). Choice of hospital: Which type of quality matters? Journal of Health Economics, 50, 230-246.

Harrington, C., Anzaldo, S., Burdin, A., Kitchener, M., \& Miller, N. (2004). Trends in state certificate of need and moratoria programs for long-term care providers. Journal of Health \& Social Policy, 19(2), 31-58.

Huang, S., \& Hirth, R. (2016). Quality rating and private-prices: Evidence from the nursing home industry. Journal of Health Economics, 50, 59-70.

National Conference of State Legislatures. (2016). CON-certificate of need laws. http://www.ncsl.org/ research/health/con-certificate-of-need-state-laws.aspx\#Program. Accessed 3 Aug 2016.

Ohlhausen, K. (2015). Certificate of need laws: A prescription for higher costs. Antitrust, 30(1), 50-54.

Ohsfeldt, R., \& Schneider, J. (2006). The business of health: The role of competition, markets and regulation. Washington, DC: AEI Press.

Pauly, M. A. (2004). Competition in medical services and the quality of care: Concepts and history. International Journal of Health Care Finance and Economics, 4, 113-130.

Phillips, C., Hawes, C., Lieberman, T., \& Koren, M. (2007). Where should momma go? Current nursing home performance measurement strategies and a less ambitious approach. BMC Health Services Research, 7, 93.

Polsky, D., David, G., Yang, J., Kinosianc, B., \& Werner, R. (2014). The effect of entry regulation in the health care sector: The case of home health. Journal of Public Economics, 110, 1-14.

Rahman, M., Galarraga, O., Zinn, J., Grabowski, D., \& Mor, V. (2016). The impact of certificate-of-need laws on nursing home and home health care expenditures. Medical Care Research and Review, 73(1), $85-105$.

Robinson, J. C., \& MacPherson, K. (2012). Payers test reference pricing and centers of excellence to steer patients to low-price and high-quality providers. Health Affairs, 31(9), 2028-2036.

Stigler, G. (1971). The theory of economic regulation. The Bell Journal of Economics and Management Science, 2(1), 3-21.

Stratmann, T., \& Wille, D. (2016). Certificate-of-need laws and hospital quality. In Mercatus Working Paper, George Mason University (September). https://www.mercatus.org/system/files/mercatus-stratmannwille-con-hospital-quality-v1.pdf. Accessed 30 Nov 2016.

Trust for America's Health. (2014). The State of Obesity. Plus reports for previous years. http://stateofobesity. org/files/stateofobesity2014.pdf. Accessed 30 Aug 2016.

Tudor, C. G. (1995). Medicaid expenditures and state responses. Health Care Financing Review, 16(3), $1-10$.

U.S. Department of Housing and Urban Development. (2015). HUD USPS Zip Code Crosswalk Files. Office of Policy Development and Research. https://www.huduser.gov/portal/datasets/usps_crosswalk.html. Accessed 1 Feb 2016.

Vaughan-Sarrazin, M., Hannan, E., Gormley, C., et al. (2002). Mortality in medicare beneficiaries following coronary artery bypass graft surgery in states with and without certificate of need regulation. JAMA, 288(15), 1859-1866.

Wiener, J., Stevenson, D., \& Goldenson, S. (1999). Controlling the supply of long-term care providers in thirteen states. Journal of Aging \& Social Policy, 10(4), 51-72. 